\title{
Sars-CoV-2 Envelope and Membrane Proteins: Structural Differences Linked to Virus Characteristics?
}

\author{
Martina Bianchi $\mathbb{D},{ }^{1}$ Domenico Benvenuto $\mathbb{D}^{2},{ }^{2}$ Marta Giovanetti $\mathbb{D}^{3},{ }^{3}$ Silvia Angeletti $\mathbb{D},{ }^{4}$ \\ Massimo Ciccozzi id, ${ }^{2}$ and Stefano Pascarella id ${ }^{1}$ \\ ${ }^{1}$ Department of Biochemical sciences "A Rossi Fanelli", Sapienza University of Rome, 00185 Rome, Italy \\ ${ }^{2}$ Unit of Medical Statistics and Molecular Epidemiology, University Campus Bio-Medico of Rome, Rome, Italy \\ ${ }^{3}$ Flavivirus Laboratory, Oswaldo Cruz Institute, Oswaldo Cruz Foundation, Rio de Janeiro, Brazil \\ ${ }^{4}$ Unit of Clinical Laboratory Science, University Campus Bio-Medico of Rome, Rome, Italy
}

Correspondence should be addressed to Stefano Pascarella; stefano.pascarella@uniroma1.it

Received 1 April 2020; Revised 23 April 2020; Accepted 7 May 2020; Published 30 May 2020

Academic Editor: Miguel A. Andrade

Copyright $\odot 2020$ Martina Bianchi et al. This is an open access article distributed under the Creative Commons Attribution License, which permits unrestricted use, distribution, and reproduction in any medium, provided the original work is properly cited.

The Coronavirus Disease 2019 (COVID-19) is a new viral infection caused by the severe acute respiratory coronavirus 2 (SARS-CoV-2). Genomic analyses have revealed that SARS-CoV-2 is related to Pangolin and Bat coronaviruses. In this report, a structural comparison between the Sars-CoV-2 Envelope and Membrane proteins from different human isolates with homologous proteins from closely related viruses is described. The analyses here reported show the high structural similarity of Envelope and Membrane proteins to the counterparts from Pangolin and Bat coronavirus isolates. However, the comparisons have also highlighted structural differences specific of Sars-CoV-2 proteins which may be correlated to the cross-species transmission and/or to the properties of the virus. Structural modelling has been applied to map the variant sites onto the predicted three-dimensional structure of the Envelope and Membrane proteins.

\section{Introduction}

COVID-19 has become a planetary emergency which is seriously threatening human health $[1,2]$. Development of effective therapeutic and prevention strategies is significantly hampered also by the lack of detailed structural information on virus proteins, although several crystallographic structures of Sars-CoV-2 proteins are now available [3-5]. In this report, a structural comparison between the Sars-CoV-2 surface proteins from different isolates with homologous proteins from closely related viruses such as those from Bat and Pangolin is described. This work has been focussed onto the Envelope (E) and Membrane (M) proteins that, along with the Spike, form the virus protein interface to the external environment. The Spike glycoprotein has been already extensively studied, and a few crystallographic structures are available in the Protein Data Bank [3-6]; consequently, this protein has not been specifically addressed within this note. Identification of local structural differences, even minimal, to the closest virus proteins may indicate the mutations that enabled Sars-CoV-2 to cross species and/or to acquire its peculiar pathogenic properties $[7,8]$. Indeed, a number of examples have been reported in the scientific literature suggesting how even single point mutations in virus proteins can significantly alter their biology and pathogenesis $[9,10]$. Therefore, comparative studies may shed light on the molecular mechanisms through which an epidemic of epizootic origin can emerge and may also suggest molecular targets for therapeutics or reverse vaccinology experiments.

\section{Material and Methods}

Nucleotide and protein sequences have been taken from GenBank [11] data repository. Blast suite [12] has been used for databank searches; Jalview [13] and MAFFT [14] have been used for multiple sequence display and alignment, respectively. Transmembrane helix prediction has been obtained by TMHMM [15], MEMSAT [16], and Protter 
Sars-CoV-2

Sars-CoV-2_isolate_SNU01

SARS-CoV-2 USA WA-UW-1588

Pangolin_CoV_MP789

Sars-CoV_ZJ01

Sars-CoV TJ01

Bat_Sars-CoV_BtKY72

Bat CoV 273/2005

Sars-CoV_Sin_WNV

\section{Sars-CoV-2}

Sars-CoV-2 isolate_SNU01

SARS-CoV-2_USA_WA-UW-1588

Pangolin_CoV_MP789

Sars-CoV_ZJ01

Sars-CoV_TJ01

Bat_Sars-CoV_BtKY72

Bat_CoV_273/2005

Sars-CoV_Sin_WNV

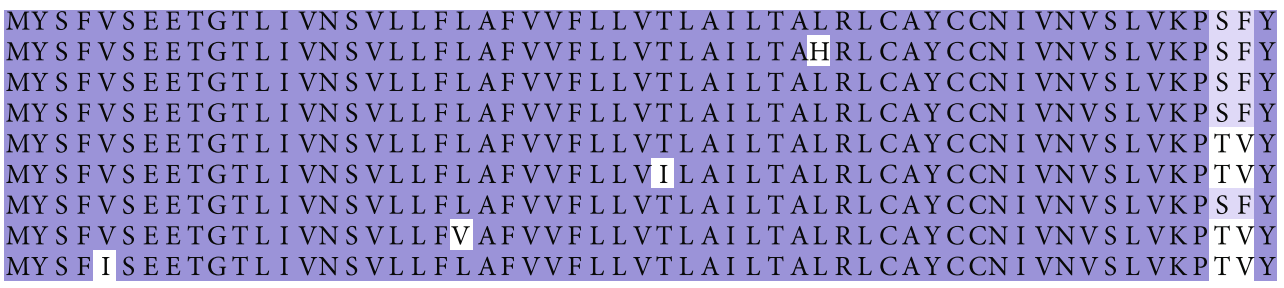

60

70

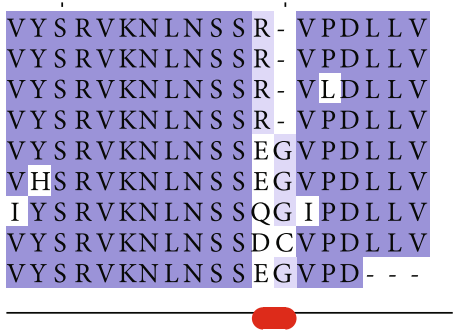

Figure 1: Multiple sequence alignment among Sars-CoV-2 Envelope protein variants and a set of the most similar homologous proteins. The sequence labelled Sars-CoV-2 corresponds to the reference sequence identified by the RefSeq code YP_009724392. Red lines below the alignment indicate the changed sites discussed in the text. Blu background denotes conserved alignment positions.

[17]. Cd-hit program [18] has been used for sequence clustering. Homology modelling relied on Swiss-Model [19], Modeller [20], or HHpred [21] and structure display and analysis on Open-Source PyMOL [22]. When necessary, I-Tasser [23] has been used as an alternative source of ab initio homology models.

\section{Results}

3.1. Databank Searches and Structure Modelling. From the GenBank repository, 797 complete genomes of Sars-CoV-2 have been collected (the full list is reported in Supplementary Data). The TblastN program has been used to extract the sequences of $\mathrm{E}$ and $\mathrm{M}$ proteins from each genome. To remove redundancy within each $\mathrm{E}$ and $\mathrm{M}$ protein set, cd-hit clustering has been applied at $100 \%$ sequence identity level: identical sequences have been assigned to the same group for which only one representative has been considered for further analysis. The Sars-CoV-2 E and M protein sets have been grouped into three and seven clusters, respectively. This finding suggests that within the 797 genomes three and seven variants of the $\mathrm{E}$ and $\mathrm{M}$ proteins can be observed, respectively. $\mathrm{E}$ and $\mathrm{M}$ homologous proteins from closely related virus have been retrieved from the GenBank using the TblastN tool.

3.2. Envelope Protein. The E protein is conserved across $\beta$-coronaviruses. Only three variants have been found in the Sars-CoV-2 E set collected. Sequence comparisons show that the Sars-CoV-2 E protein from the reference genome (RefSeq code YP_009724392) is identical to the sequences from Pangolin CoV MP798 and Bat CoV CoVZXC21, CoVZC45, and RaTG13 isolates. The multiple sequence alignment reported in Figure 1 demonstrates that a distinguishing feature of Sars-2-CoV E variants is the presence of
Arg at position 69 that substitutes Glu, Gln, Asp in other homologous Sars-CoV E proteins. This site is followed by a deletion in position 70 corresponding to Gly or Cys in the other proteins. Sars-CoV-2 E sequences differ from the homologous proteins also at positions 55-56, where the dyad Ser-Phe replaces Thr-Val (except in Bat coronavirus isolate BtKY72, accession code KY352407). Variants of the Sars-CoV-2 E protein differ at positions 37 and 72 where His substitutes a Leu and Leu replaces a conserved Pro, respectively. The size of each Envelope variant cluster is reported in Table 1 along with accession codes and definitions of the isolates. A homology model of the E protein has been built with Modeller using as a template the pentameric ion channel structure of Sars-CoV protein identified by the PDB code $5 X 29$. This sequence shares $91 \%$ identity to Sars-CoV$2 \mathrm{E}$ protein and covers the segment encompassed by positions 8-65. Figure 2 displays the structure of the homology model of the Sars-CoV-2 E protein assembled as a pentameric viroporin-like protein. Figure 2 displays also the position of the variant sites onto the three-dimensional model. Prediction of the transmembrane helices is difficult in a short protein. Therefore, transmembrane topology cannot be assigned reliably. Likewise, experiments have not clarified definitively which portions of the E protein are exposed to the external or internal side of the virus membrane [24].

3.3. Membrane Glycoprotein. The M glycoprotein is conserved across the $\beta$-coronaviruses. However, seven variants of Sars-CoV-2 M protein were identified in the collected set, while only three variants were observed for the E protein (Figure 3). The multiple sequence alignment shows a remarkable similarity ( $98 \%$ identity) among the Sars-CoV-2 $M$ variants and the sequences from Bat and Pangolin isolates. However, a difference at the N-terminal position (Figure 3) can be observed: the insertion of a Ser residue at position 4 
TABLE 1: Size of the variant clusters of the Sars-CoV-2 Envelope and Membrane proteins.

\begin{tabular}{lccc}
\hline Variant & Cluster size (no. of sequences) & Accession code & Definition \\
\hline $\begin{array}{l}\text { Envelope } \\
\text { YP_009724392 (reference) }\end{array}$ & 795 & & Korea/SNU01/2020 \\
\hline His37 & 1 & MT03980 & USA/WA-UW-1588/2020 \\
\hline Leu72 & 1 & MT293206 & \\
\hline Membrane & & & CHN/Wuhan_IME-BJ07/2020 \\
YP_009724393 (reference) & 773 & USA/SC_3572/2020 \\
\hline Ser2 & 1 & MT291836 & VIE/NIHE/2020 \\
\hline Gly3 & 1 & MT127115 & USA/WA-UW-1297/2020 \\
\hline Val57, Arg89 & 1 & MT293184 & USA/WA-UW-1735/2020 \\
\hline lle70 & & MT326166 & USA/WA-UW-1591/2020 \\
\hline Ser85 & 3 & MT293211 & USA/WA-UW-1753/2020 \\
\hline & & MT326167 & USA/WA-UW-1775/2020 \\
Met175 & 1 & MT326093 & USA/WA-UW-194/2020 \\
\hline
\end{tabular}

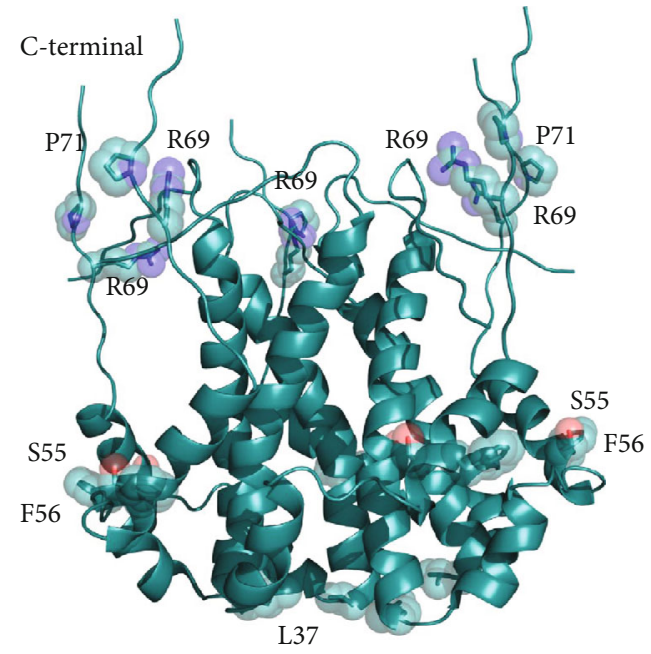

(a)

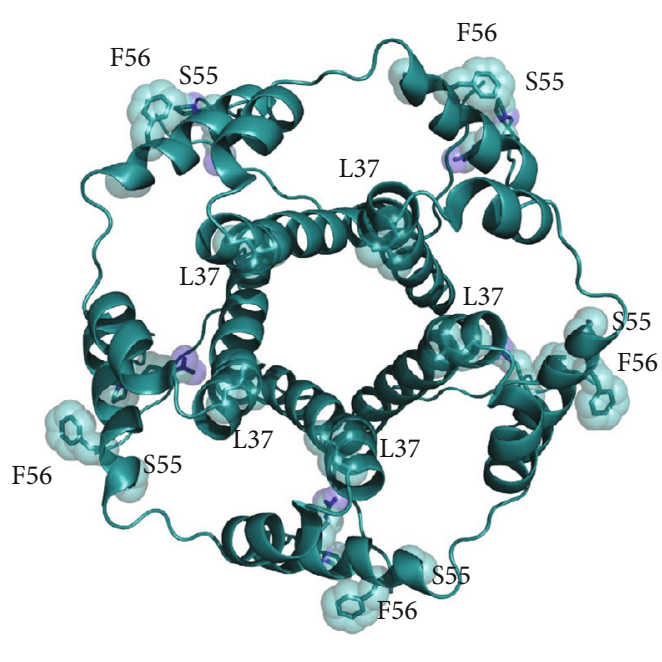

(b)

FIGURE 2: Three-dimensional model of the viroporin-like tetrameric assembly of the E protein from Sars-CoV-2 represented as a cartoon model. Residues corresponding to the mutated sites indicated in Figure 1 are displayed as transparent space-filling spheres and labelled with the amino acid one-letter code. The C-terminal segments of the model are reported for completeness. However, they convey no structural information due to lack of a corresponding segment in the structural template used in homology modelling. Structure in panel (b) is rotated by approximately $180^{\circ}$ along the $x$ axis with respect to the orientation shown in panel (a).

of human Sars-CoV-2 seems to be a unique feature of this protein. In the corresponding position, the RaTG13 Bat M protein displays a deletion, while Bat CoVZXC21, CoVZC45, and Pangolin MP789 proteins have an Asp residue. The seven $M$ protein variants differ at positions $2,3,57,70,85$, 89 , and 175. The size of each Membrane variant cluster is reported in Table 1 along with accession codes and definitions of the isolates. Noteworthy, the protein from the SarsCoV-2 NIHE isolate (accession code MT127115) possesses an Arg instead of a conserved Gly at position 89 (Figure 3). The mutation occurs within a predicted transmembrane helix and, if confirmed, may have a significant impact on the protein properties (Figure 3 ).

The three-dimensional model of the $\mathrm{M}$ protein has been taken from the I-Tasser server (code QHD43419) as other methods failed to find any suitable template. However, it should be mentioned that HHpred found a weak local affinity, albeit below the statistical significance level, to 4 N31, a peptidase-like protein from Streptococcus pyogenes essential for pilus polymerisation. Figure 4 displays the positions of the variant sites onto the model structure. This model has been predicted by ab initio techniques. Therefore, it should 
10

20

30

40

50

Sars- CoV-2 Sars- CoV-2 NIHE

Sars_CoV-2_Wuhan_IME-BJO7

Sars- CoV-2_WA-UW-1753

Sars- CoV-2_WA-UW-1775

SARS-CoV-2_USA_SC_3572

Bat CoV RaTG13

Bat_CoVZXC21

Pangolin_CoV_MP789

Bat_CoV_GX2013,

Bat_CoV_As6526
Sars_CoV-2_Wa9-UW6

Sars-CoV_GD322

MADSNGT I TVEELKKLLEQWNLV I GF LFLTW I CLLQFAYANRNR F LY I I KL I F LWL LW MADSNGT I TVEELKKLLEQWNLV I GFLFLTWI CLLQFAYANRNRFLY I I KL I F LWLLW MADSNGT I TVEELKKLLEQWNLV I GFLFLTWI CLLQFAYANRNR F LY I I KL I F LWLVW MSDSNGT I TVEELKKLLEQWNLV I GFLFLTWI CLLQFAYANRNRFLY I I KL I FLWLLW MADSNGT I TVEELKKLLEQWNLV I GFLFLTWI CLLQFAYANRNR F LY I I KL I F LWLLW MADSNGT I TVEELKKLLEQWNLV I GFLFLTWI CLLQFAYANRNRFLY I I KL I FLWLLW MAGSNGT I TVEELKKLLEQWNLV I GFLFLTWI CLLQFAYANRNRFLY I I KL I F LWLLW MAD-NGT I TVEELKKLLEQWNLV I GFLFLTWI CLLQFAYANRNRFLY I I KL I F LWLLW MSGDNGT I TVEELKKLLEQWNLV I GFLFLTW I CLLQFAYANRNRFLY I I KL I FLWLLW MSGDNGT I TVEELKKLLXQWNLV I GFLFLTWI CLLQFAYANRNRFLY I I KL I FLWLLW MAD- NGT I TVEELKQ LLEQWNLV I GF LF LAW I MLLQFAY SNRNR F LY I I K LVF LWL LW MAD-NGT I TVEELKQLLEQWNLV I GFLFLAWIMLLQFAY SNRNRF LY I I KLVF LWLLW MAD-NGT I TVEELKQ LLEQWNLV I GF LF LAW I MLLQFAY SNRNR F LY I I K LVF LWL LW TMH

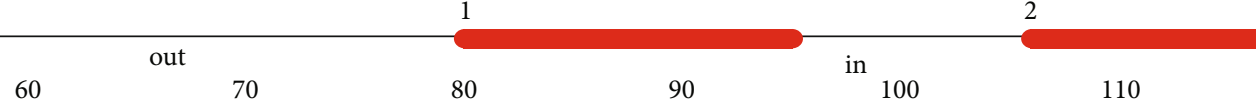

Sars- CoV-2

Sars_CoV-2_Wa9-UW6

Sars- CoV-2 NIHE

Sars_CoV-2_Wuhan_IME-BJO7

Sars- CoV-2 WA-UW-1753

Sars- CoV-2_WA-UW-1775

SARS-CoV-2_USA_SC_3572

Bat_CoV_RaTG13

Bat_CoVZ̄XC21

Pangolin_CoV_MP789

Bat_CoV_GX2013,

Bat CoV As6526

Sars-CoV_GD322

PVT LACFVLAAVYR I NWI T GG I A I AMACLVGLMWLSY F I A SFR LFARTR SMWSFNPET $P V T L A C F V L A A \mid Y R$ I NWI T GG I A I AMACLVGLMWLSYF I ASFRLFARTR SMWSFNPET PVT LACFVLAAVYR I NWI TGG I A I AMACLVRLMWLSYF I A SFRLFARTR SMWSFNPET PVTLACFVLAAVYR I NWITGGIA I AMACLVGLMWLSYF I ASFRLFARTR SMWSFNPET $P \vee T L A C F \vee L A A V Y R$ I NWI T GG I A I AMSCLVGLMWL SYF I A SFRLFARTR SMWSFNPET PVTLACFVLAAVYR I NWITGG I A I AMACLVGLMWLSYFIASFRLFARTR SMWSFNPET $P V T L A C F V L A A V Y R$ I NWITGGIA I AMACLVGLMWLSYFIASFRLFARTR SMWSFNPET PVTLACFVLAAVYR I NWITGGIA I AMACLVGLMWLSYF I A SFRLFARTR SMWSFNPET $P \vee T L A C F V L A A V Y R$ I NWI TGG I A I AMACLVGLMWLSYFIASFRLFARTR SMWSFNPET $P \vee T L A C F \vee L A A V Y R$ I NWI T GG I A I AMACLVGLMWL SYF I A SFRLFARTR SMWSFNPET $P \vee T L A C F V L A A V Y R$ I NWVT GGIA I AMACIVGLMWLSYFVASFRLFARTR SMWSFNPET PVT LACFVLAAVYR I NWVT GG I A I AMACIVGLMWLSYFVASFRLFARTR SMWSFNPET PVT LACFVLAAVYR I NWVTGGIA I AMAC I VGLMWLSYFVASFRLFARTR SMWSFNPET

$\mathrm{TMH}$

$\begin{array}{lccccc}\text { out } & 140 & 150 & \text { in } & 160 & 170\end{array}$

Sars- CoV-2

Sars_CoV-2_Wa9-UW6

Sars- CoV-2 NIHE

Sars_CoV-2_Wuhan_IME-BJO7

Sars- CoV-2_WA-UW-1753

Sars- CoV-2_WA-UW-1775

SARS-CoV-2_USA_SC_3572

Bat_CoV_RaTG13

Bat_CoVZ̄XC21

Pangolin_CoV_MP789

Bat_CoV_GX2013,

Bat CoV As6526

Sars-CoV_GD322
N I L LNVP LHGT I LTRPLLESELVI GAVI LRGHLR I AGHHLGRCDIKDLPKEITVATSR N I LLNVP LHGT I LTRPLLESELVIGAVI LRGHLR IAGHHLGRCDIKDLPKEITVATSR N I LLNVPLHGT I LTRPLLESELVIGAVILRGHLR IAGHHLGRCDIKDLPKEITVATSR N I LLNVPLHGT I LTRPLLESELVIGAVILRGHLR I AGHHLGRCDIKDLPKEITVATSR N I LLNVPLHGT I LTRPLLESELVIGAVILRGHLR I AGHHLGRCDIKDLPKEITVATSR N I L LNVP LHGT I LTRPLLESELVIGAVI LRGHLR I AGHHLGRCDIKDLPKEITVATSR N I LLNVPLHGT I LTRPLLESELVIGAVILRGHLR I AGHHLGRCDIKDLPKEITVATSR N I LLNVPLHGT I LTRPLLESELVIGAVILRGHLR I AGHHLGRCDIKDLPKEITVATSR N I LLNVPLHGT I LTRPLLESELVIGAVI LRGHLRIAGHHLGRCDIKDLPKEITVATSR N I LLNVPLHGT I LTRPLLESELVIGAVI LRGHLR I AGHHLGRCDIKDLPKEITVATSR N I LLNVPLRGT I LTRPLMESELVIGAV I I RGHLRMAGHSLGRCDIKDLPKE ITVATSR N I LLNVPLRGT I VTRPLLESELVI GAV I I RGHLRMAGHSLGRCDIKDLPKE ITVATSR N I LLNVPLRGT IVTRP LMESELVIGAVI I RGHLRMAGHSLGRCDIKDLPKEITVATSR
Sars- CoV-2

Sars_CoV-2_Wa9-UW6

Sars- CoV-2_NIHE

Sars_CoV-2_Wuhan_IME-BJO7

Sars- CoV-2 WA-UW-1753

Sars- CoV-2_WA-UW-1775

SARS-CoV-2_USA_SC_3572

Bat_CoV_RaTG13

Bat_CoVZ̄XC21

Pangolin_CoV_MP789

Bat_CoV_GX2013,

Bat_CoV_As6526

Sars-CoV_GD322

TMH

180

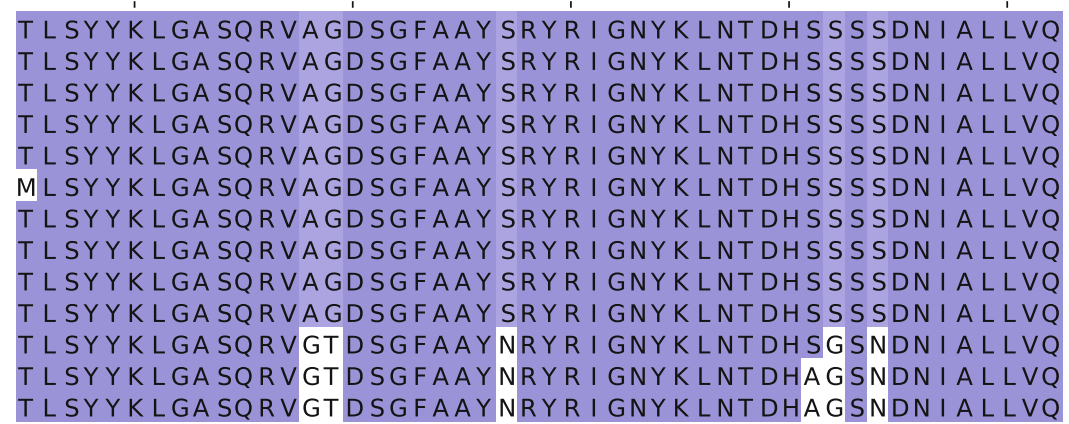

TMH

FIGURE 3: Multiple sequence alignment among Sars-CoV-2 M protein variants and a set of most similar homologous proteins. The sequence label Sars-CoV-2 indicates the reference sequence identified by the RefSeq code YP_009724393. Red box indicates the variant sites at the N-terminal discussed in the text. Numbered red bars under the multiple alignment mark the prediction of transmembrane helices. The location of the connect loop with respect to the virion surface is indicated as "in" or "out". Blu background denotes conserved alignment positions. 


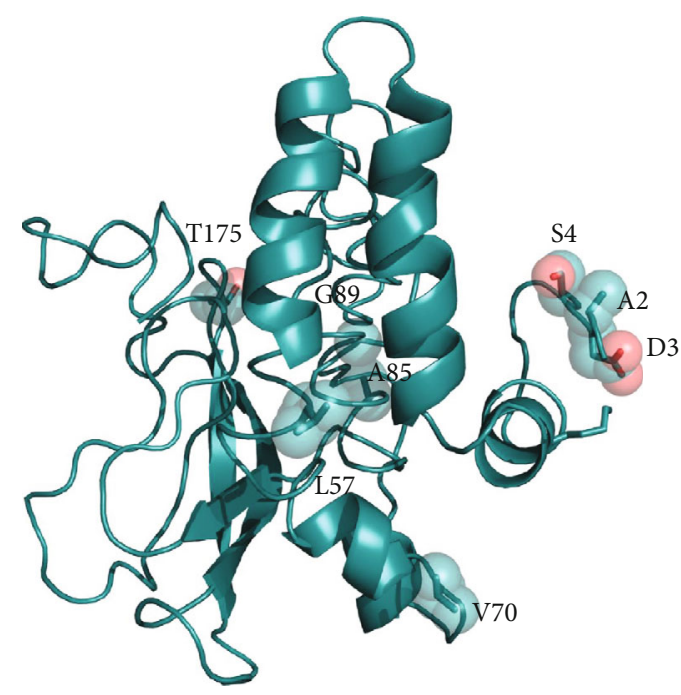

FIGURE 4: I-Tasser model of the Membrane protein represented as cartoon model. Variant positions are displayed as transparent space-filling spheres and labelled with the amino acid one-letter code.

be considered with great caution and should be treated as a low-resolution approximation of the real structure. According to the prediction of the transmembrane helix topology, the $\mathrm{N}$ - and $\mathrm{C}$-terminal portions of the $\mathrm{M}$ protein are exposed outside and inside the virus particle, respectively (Figure 4).

\section{Discussion}

Previous studies pointed out that $\mathrm{E}$ and $\mathrm{M}$ proteins could be important for viral entry, replication, and particle assembly within the human cells $[24,25]$. According to the accepted theories, the current COVID-19 pandemic has been caused by the cross-species transmission of a $\beta$-coronavirus normally hosted by Bats and, perhaps, Pangolin to humans $[3,26]$. In this paper, $E$ and $M$ proteins from 797 SarsCoV-2 genomes have been compared to the counterparts taken from the most closely related virus also to evaluate the potential role of amino acid mutations in the epizootic origin of COVID-19. E protein is a minor component of the virus membrane though it is deemed to be important for many stages of virus infection and replication [24, 25]. Sequence comparison has shown that this protein is identical to the counterparts of specific Bat and Pangolin coronavirus isolates, even though the Sars-CoV-2 sequence seems to possess specific modifications and characteristics with respect to other Sars CoVs. In particular, Arg69, a positively charged amino acid, replaces Glu or Gln residues, negatively charged and neutral, respectively, in the homologous $\mathrm{CoV}$ proteins. Moreover, a deletion specific to Sars-CoV-2 proteins flanks this position. Unfortunately, it is not possible to predict reliably whether the sites of these modifications are exposed to the internal or external side of the membrane. In any case, the substitution and the deletion appear a rather drastic change and may have a significant impact on conformational properties and possibly on protein-protein interactions. Further structural studies are needed. However, it may be hypothesized that these changes can also affect the olig- omerization process necessary to form a transmembrane ion channel.

It has been demonstrated that $\mathrm{M}$ protein is more prevalent within the virus membrane, and it is deemed to be important for the budding process of coronaviruses. Indeed, during the process of virus particle assembly, this protein interacts with the Nucleocapsid, Envelope, Spike, and Membrane glycoprotein itself [25]. Moreover, in Alphacoronaviruses, it has been demonstrated that this protein cooperates with the Spike during the cell attachment and entry [27]. Therefore, mutations occurring at the N-terminus region, which is exposed to the virus surface, could play a key role in the host cell interaction.

In conclusion, the analyses here reported show the structural similarity of $\mathrm{E}$ and $\mathrm{M}$ proteins to the counterparts from Pangolin and Bat coronavirus isolates. At the same time, comparisons have highlighted structural differences specific of Sars-CoV-2 proteins which may be correlated to the cross-species transmission and/or to the properties of the virus. Although further studies are needed, it is clear that these amino acid variations have been important for the virus evolutionary history, and the results may hint at how similar mutations within the coronavirus family can lead in the next years to other epizootic epidemic events similar to the one that we are experiencing these days.

\section{Data Availability}

All sequence data are available in the GenBank repository. The complete list is available in the Supplementary Materials.

\section{Conflicts of Interest}

The authors declare that there is no conflict of interest regarding the publication of this paper. 


\section{Acknowledgments}

This work has been in part funded by a grant to SP from Sapienza University of Rome (RP11916B74B27C4D).

\section{Supplementary Materials}

The supplementary data consist of an Excel file containing the list of the 797 Sars-CoV-2 genomes retrieved from GenBank and analysed in the article. For each genome, the sequence header is displayed as found in the GenBank. The header reports the accession code in the first field of each line after the ">" character. (Supplementary Materials)

\section{References}

[1] D. Benvenuto, M. Giovanetti, A. Ciccozzi, S. Spoto, S. Angeletti, and M. Ciccozzi, "The 2019-new coronavirus epidemic: evidence for virus evolution," Journal of Medical Virology, vol. 92, no. 4, pp. 455-459, 2020.

[2] C.-C. Lai, T.-P. Shih, W.-C. Ko, H.-J. Tang, and P.-R. Hsueh, "Severe acute respiratory syndrome coronavirus 2 (SARSCoV-2) and coronavirus Disease-2019 (COVID-19): the epidemic and the challenges," International Journal of Antimicrobial Agents, vol. 55, no. 3, article 105924, 2020.

[3] D. Benvenuto, M. Giovanetti, M. Salemi et al., "The Global Spread of 2019-NCoV: A Molecular Evolutionary Analysis," Pathogens and Global Health, vol. 114, no. 2, pp. 64-67, 2020.

[4] A. C. Walls, Y.-J. Park, M. A. Tortorici, A. Wall, A. T. McGuire, and D. Veesler, "Structure, Function, and Antigenicity of the SARS-CoV-2 Spike Glycoprotein," Cell, vol. 181, no. 2, pp. 281-292.e6, 2020.

[5] D. Wrapp, N. Wang, K. S. Corbett et al., "Cryo-EM Structure of the 2019-NCoV Spike in the Prefusion Conformation," Science, vol. 367, no. 6483, pp. 1260-1263, 2020.

[6] R. Yan, Y. Zhang, Y. Li, L. Xia, Y. Guo, and Q. Zhou, "Structural Basis for the Recognition of SARS-CoV-2 by Full-Length Human ACE2," Science, vol. 367, no. 6485, pp. 1444-1448, 2020.

[7] S. Angeletti, D. Benvenuto, M. Bianchi, M. Giovanetti, S. Pascarella, and M. Ciccozzi, "COVID-2019: the role of the Nsp2 and Nsp3 in its pathogenesis," Journal of Medical Virology, vol. 92, no. 6, pp. 584-588, 2020.

[8] W. Ji, W. Wang, X. Zhao, J. Zai, and X. Li, "Cross-species transmission of the newly identified coronavirus 2019-NCoV," Journal of Medical Virology, vol. 92, no. 4, pp. 433-440, 2020.

[9] N. M. André, B. Cossic, E. Davies, A. D. Miller, and G. R. Whittaker, "Distinct mutation in the feline coronavirus spike protein cleavage activation site in a cat with feline infectious peritonitis-associated Meningoencephalomyelitis," Journal of Feline Medicine and Surgery Open Reports, vol. 5, no. 1, 2019.

[10] Y. Sakai, K. Kawachi, Y. Terada, H. Omori, Y. Matsuura, and W. Kamitani, "Two-amino acids change in the Nsp4 of SARS coronavirus abolishes viral replication," Virology, vol. 510, pp. 165-174, 2017.

[11] D. A. Benson, M. Cavanaugh, K. Clark et al., "GenBank," Nucleic Acids Research, vol. 46, no. D1, pp. D41-D47, 2018.

[12] S. Altschul, T. L. Madden, A. A. Schäffer et al., "Gapped BLAST and PSI-BLAST: a new generation of protein database search programs," Nucleic Acids Research, vol. 25, no. 17, pp. 3389-3402, 1997.

[13] A. M. Waterhouse, J. B. Procter, D. M. A. Martin, M. Clamp, and G. J. Barton, "Jalview version 2-a multiple sequence alignment editor and analysis workbench," Bioinformatics, vol. 25, no. 9, pp. 1189-1191, 2009.

[14] K. Katoh and D. M. Standley, "MAFFT multiple sequence alignment software version 7: improvements in performance and usability," Molecular Biology and Evolution, vol. 30, no. 4, pp. 772-780, 2013.

[15] Y. Chen, P. Yu, J. Luo, and Y. Jiang, "Secreted protein prediction system combining CJ-SPHMM, TMHMM, and PSORT," Mammalian Genome, vol. 14, no. 12, pp. 859-865, 2003.

[16] L. J. McGuffin, K. Bryson, and D. T. Jones, "The PSIPRED protein structure prediction server," Bioinformatics, vol. 16, no. 4, pp. 404-405, 2000.

[17] U. Omasits, C. H. Ahrens, S. Müller, and B. Wollscheid, "Protter: interactive protein feature visualization and integration with experimental proteomic data," Bioinformatics, vol. 30, no. 6, pp. 884-886, 2014.

[18] L. Fu, B. Niu, Z. Zhu, S. Wu, and W. Li, "CD-HIT: accelerated for clustering the next-generation sequencing data," Bioinformatics, vol. 28, no. 23, pp. 3150-3152, 2012.

[19] A. Waterhouse, M. Bertoni, S. Bienert et al., "SWISS-MODEL: homology Modelling of protein structures and complexes," Nucleic Acids Research, vol. 46, no. W1, pp. W296-W303, 2018.

[20] B. Webb and A. Sali, "Protein structure modeling with MODELLER," Methods in Molecular Biology, vol. 1654, pp. 39-54, 2017.

[21] L. Zimmermann, A. Stephens, S.-Z. Nam et al., "A completely Reimplemented MPI bioinformatics toolkit with a new HHpred server at its Core," Journal of Molecular Biology, vol. 430, no. 15, pp. 2237-2243, 2018.

[22] L. L. C. Schrodinger, The AxPyMOL Molecular Graphics System, Version 2.0, Schrödinger, LLC., 2015.

[23] J. Yang, R. Yan, A. Roy, D. Xu, J. Poisson, and Y. Zhang, "The I-TASSER suite: protein structure and function prediction," Nature Methods, vol. 12, no. 1, pp. 7-8, 2015.

[24] D. Schoeman and B. C. Fielding, "Coronavirus envelope protein: current knowledge," Virology Journal, vol. 16, no. 1, p. 69, 2019.

[25] E. A. J. Alsaadi and I. M. Jones, "Membrane binding proteins of coronaviruses," Future Virology, vol. 14, no. 4, pp. 275286, 2019.

[26] R. Lu, X. Zhao, J. Li et al., "Genomic characterisation and epidemiology of 2019 novel coronavirus: implications for virus origins and receptor binding," The Lancet, vol. 395, no. 10224, pp. 565-574, 2020.

[27] A. Naskalska, A. Dabrowska, A. Szczepanski, A. Milewska, K. P. Jasik, and K. Pyrc, "Membrane protein of human coronavirus NL63 is responsible for interaction with the adhesion receptor," Journal of Virology, vol. 93, no. 19, pp. 455-459, 2019. 